SNUTP 97-085

hep-th/9706184

\title{
Second Virial Coefficient of Anyons without Hard Core
}

\author{
Chanju Kim* \\ Center for Theoretical Physics \\ Seoul National University \\ Seoul, 151-742, Korea
}

\begin{abstract}
We calculate the second virial coefficient of anyons whose wave function does not vanish at coincidence points. This kind of anyons appear naturally when one generalizes the hard-core boundary condition according to self-adjoint extension method in quantum mechanics, and also when anyons are treated field theoretically by applying renormalization procedure to nonrelativistic ChernSimons field theory. For the anyons which do not satisfy hard-core boundary condition, it is argued that the other scale-invariant limit is more relevant in high-temperature limit where virial expansion is useful. Furthermore, the cusp existing at the bosonic point for hard-core anyons disappears in general; instead it is shown that a new cusp is generated at the fermionic point. A physical explanation is given.
\end{abstract}

PACS number: 11.10.Kk, 05.30.-d, 71.10.Pm

*cjkim@ctp.snu.ac.kr 
In this letter we would like to calculate the second virial coefficient of a system of anyons [1] obtained by generalizing the usual hard-core boundary condition at coincidence points according to the self-adjoint extension method [2], and discuss its interesting physical properties. To motivate our calculation, let us briefly summarize the present status.

Anyons are particles exhibiting fractional statistics characterized by a statistical parameter $\alpha$ which interpolates between bosonic and fermionic cases. Quantum mechanically, they can be regarded as flux-charge composites and their interaction is essentially AharonovBohm type [河]. From the field theoretic point of view, they can be obtained if the Schrödinger field is coupled to a Chern-Simons gauge field [4.5.5. The connection of these two descriptions is now well established and an interesting subject here is the relation between the method of self-adjoint extension in quantum mechanics and the regularization-renormalization procedure in field theory where $|\phi|^{4}$-type contact interaction is necessarily introduced [6 9]. Recently, an all-order analysis has been carried out to establish the precise correspondence and the role of contact term has been emphasized in ref. [9].

In the literature, regularity of wave functions is often assumed and it leads to the hardcore boundary condition [1]. Although this hard-core case is conceptually simple and easier to treat, there is no a priori reason to assume such a boundary condition in general; a real system under study should determine the relevant boundary condition eventually. If we relax the regularity requirement, allowing wave functions to diverge at coincidence points according to the method of self-adjoint extension, we get a one-parameter family of boundary conditions which in general introduces a scale in the theory. (The corresponding anyons were called "colliding anyons" [10.) The scale invariance of the theory is restored only in the limit that the scale parameter goes to either zero or infinity. Field theoretically, when the strength of the $|\phi|^{4}$-interaction is equal to the critical value $\lambda= \pm \frac{2 \pi|\alpha|}{m} \equiv \pm \lambda_{0}$, the field theory becomes ultraviolet finite and scale invariant [9]. These critical values have some more interesting properties. The repulsive case $\lambda=\lambda_{0}$ corresponds to the usual hard-core boundary condition, while in the attractive case $\lambda=-\lambda_{0}$, it is known that the classical theory admits static soliton solutions satisfying a self-dual equation [5]. Also, for $\lambda \rightarrow-\lambda_{0}$, 
after performing renormalization, one can relate the renormalized coupling $\lambda_{\text {ren }}$ to a nonzero finite value of the self-adjoint extension parameter in a specific way [11,9].

Since the free anyon gas has not been exactly solved, one usually resorts to the virial expansion to study thermodynamic properties in high-temperature low-density limit. For example, the second virial coefficient was calculated for anyons with hard core in ref. [12 [15]. Here an interesting fact is that, according to recent analyses [11,9], $\lambda_{\text {ren }}=\lambda_{0}$ (hard-core case) is an infrared fixed point, while $\lambda_{\text {ren }}=-\lambda_{0}$ is an ultraviolet fixed point, with $\lambda_{\text {ren }}$ flowing from $\lambda_{0}$ to $-\lambda_{0}$ as the renormalization scale increases. This implies that the virial expansion for usual anyons with hard core is not much relevant for the case of colliding anyons in hightemperature limit where the virial expansion is useful. Therefore it may be interesting to calculate the second virial coefficient for anyons with an arbitrary boundary condition and study the behavior as the scale changes. Indeed, we will see that the result of hard-core case does not represent a typical behavior of the other cases. For example, the cusp existing at bosonic point $\alpha=0$ in hard-core case is a very special feature of scale-invariant limit which does not occur in general; instead a new cusp is generated at the fermionic point $|\alpha|=1$ except for the hard core case. We will give a physical interpretation of this result later.

Here we should mention earlier works [16, 17] which have a partial overlap with this paper. They calculated the second virial coefficient for the case that the system has a bound state. However they did not pay much attention to physical properties for general boundary conditions except at the fixed point, $\lambda_{\text {ren }}=-\lambda_{0}$. Also, we believe that our method is simpler and physically more transparent.

Let us start with the Hamiltonian of a system of free anyons with mass $m$ and statistical parameter $\alpha$ in bosonic description

$$
H=\sum_{n} \frac{1}{2 m}\left(\mathbf{p}_{n}-\alpha \mathbf{A}_{n}\right)^{2},
$$

where

$$
A_{n}^{i} \equiv \epsilon^{i j} \sum_{m(\neq n)} \frac{x_{n}^{j}-x_{m}^{j}}{\left|\mathbf{r}_{n}-\mathbf{r}_{m}\right|^{2}}
$$


is the Aharonov-Bohm-type vector potential and $\mathbf{r}=\left(x_{n}^{1}, x_{n}^{2}\right)$ denotes the position of the $n$-th particle. As mentioned above, this Hamiltonian does not specify the system completely and one has to give a suitable boundary conditions when particle positions coincide; we will give the form later.

In two dimensions, the second virial coefficient $B(T)$ is given by 18

$$
B(T)=A\left(\frac{1}{2}-Z_{2} / Z_{1}^{2}\right)
$$

where $A$ is the area of the system and $Z_{1}$ and $Z_{2}$ are the one-body and two-body partition functions, respectively. They are calculated as

$$
\begin{aligned}
& Z_{1}=A \lambda_{T}^{2}, \\
& Z_{2}=2 A \lambda_{T}^{2} Z_{\mathrm{rel}},
\end{aligned}
$$

where $\lambda_{T}=\left(2 \pi / m k_{B} T\right)^{1 / 2}$ is the thermal wavelength and $Z_{\text {rel }}=$ Tre $e^{-\beta H_{\text {rel }}}$ is the single partition function of the relative dynamics with

$$
\begin{aligned}
H_{\mathrm{rel}} & =\frac{1}{m}(\mathbf{p}-\alpha \mathbf{A})^{2}, \\
A^{i} & \equiv \frac{\epsilon^{i j} x^{j}}{r^{2}}, \quad(r \equiv|\mathbf{r}|) .
\end{aligned}
$$

After the separation of variables $\psi(\mathbf{r})=e^{i n \theta} R_{n}(r)$, the Schrödinger equation for $H_{\text {rel }}$ reads

$$
\frac{1}{m}\left[-\frac{1}{r} \frac{d}{d r} r \frac{d}{d r}+\frac{(n+\alpha)^{2}}{r^{2}}\right] R_{n}(r)=E R_{n}(r) \equiv \frac{k^{2}}{m} R_{n}(r),
$$

where $n$ is even since we are working in bosonic description. Also, since $\alpha$ enters (6) only through the combination $n+\alpha$, we may restrict $\alpha$ to the interval $[-1,1]$ without loss of generality in considering the energy spectrum [1]. Equation (6) is nothing but the Bessel equation and the general solution is of the form

$$
R_{n}(r)=A J_{|n+\alpha|}(k r)+B J_{-|n+\alpha|}(k r)
$$

where $A, B$ are constants. For $n \neq 0$ the constant $B$ must be chosen to be zero for square integrability, but for $n=0$ ( $s$-wave case) arbitrary $A$ and $B$ are allowed. This leads to an above-mentioned one-parameter family of boundary conditions for $s$-wave [19], 


$$
\lim _{r \rightarrow 0}\left\{r^{|\alpha|} R_{0}(r)-\frac{\sigma}{\kappa^{2|\alpha|}} \frac{\Gamma(1+|\alpha|)}{\Gamma(1-|\alpha|)} \frac{d}{d\left(r^{|2 \alpha|}\right)}\left[r^{|\alpha|} R_{0}(r)\right]\right\}=0,
$$

with the corresponding solution given by

$$
R_{0}(r)=(\text { const. })\left[J_{|\alpha|}(k r)+\sigma\left(\frac{k}{\kappa}\right)^{2|\alpha|} J_{-|\alpha|}(k r)\right],
$$

where $\sigma= \pm 1$ and $\kappa$ is a scale introduced by the boundary condition. If $\kappa \rightarrow \infty$ with $\sigma=+1$ we recover the hard-core boundary condition $\psi(0)=0$. In addition to the solution (9), if $\sigma=-1$, there is a bound state satisfying (8),

$$
R_{0}(r)=(\text { const. }) K_{|\alpha|}(\kappa r)
$$

with energy $E_{B}=-\kappa^{2} / m$. (Thus the parameter $\kappa$ in the boundary condition appears in the bound state energy.) To proceed, we introduce a circular boundary at radius $R$ and assume $R_{0}(R)=0$ as usual [12]. The raduis $R$ will be taken to be infinity eventually. Then the allowed energies are

$$
\begin{aligned}
& E_{n, s}=\frac{k_{n, s}^{2}}{m} \quad(n \neq 0), \\
& E_{0, s}=\frac{\tilde{k}_{s}^{2}}{m} \quad(n=0),
\end{aligned}
$$

where $k_{n, s} R$ is the $s$-th zero of $J_{|n+\alpha|}(k R)=0$ and $\tilde{k}_{s} R$ is the $s$-th zero of (9), i.e.,

$$
J_{|\alpha|}(k R)+\sigma\left(\frac{k}{\kappa}\right)^{2|\alpha|} J_{-|\alpha|}(k R)=0 .
$$

Hence, compared with the hard-core case, only $s$-wave energy spectrum is changed.

From Eqs. (3) and (4), the second virial coefficient can then be written as

$$
B(T)=B_{0}(T)-2 \lambda_{T}^{2}\left\{e^{-\beta E_{B}} \theta(-\sigma)+\lim _{R \rightarrow \infty} \sum_{s=0}^{\infty}\left[e^{-\beta \frac{\tilde{k}_{s}^{2}}{m}}-e^{-\beta \frac{k_{0, s}^{2}}{m}}\right]\right\},
$$

\footnotetext{
${ }^{1}$ If $\sigma=-1$, formally we obtain $\psi(0)=0$ when $\kappa \rightarrow \infty$ but it does not correspond to the usual hard-core limit. Actually, the system is not bounded from below because it has a bound state with negative infinite energy as seen in (10) below [19].
} 
where $\beta=1 / k_{B} T$ and $B_{0}(T)$ is the second virial coefficient for hard-core anyons calculated in ref. $12 \pi$,

$$
B_{0}(T)=\lambda_{T}^{2}\left(-\frac{1}{4}+|\alpha|-\frac{\alpha^{2}}{2}\right) .
$$

To perform the summation in (13) in the limit $R \rightarrow \infty$, we rewrite (12) using $J_{-|\alpha|}(x)=$ $\cos \pi \alpha J_{|\alpha|}(x)-\sin \pi|\alpha| N_{|\alpha|}(x)$

$$
\cos \eta(k) J_{|\alpha|}(k R)-\sin \eta(k) N_{|\alpha|}(k R)=0,
$$

where $\eta(k)$ is defined as

$$
\tan \eta(k)=\frac{\sigma\left(\frac{k}{\kappa}\right)^{2|\alpha|} \sin \pi|\alpha|}{1+\sigma\left(\frac{k}{\kappa}\right)^{2|\alpha|} \cos \pi \alpha} .
$$

Then, from the asymptotic behavior of Bessel functions [20],

$$
\begin{aligned}
J_{|\alpha|}(x) & =\sqrt{\frac{2}{\pi x}} \cos \left[x-\left(|\alpha|+\frac{1}{2}\right) \frac{\pi}{2}\right]+\cdots, \\
N_{|\alpha|}(x) & =\sqrt{\frac{2}{\pi x}} \sin \left[x-\left(|\alpha|+\frac{1}{2}\right) \frac{\pi}{2}\right]+\cdots,
\end{aligned}
$$

it is easy to see that, for any $\epsilon>0$, there exists an integer $s_{0}$ independent of $R$ such that

$$
\left|\cos \left[\tilde{k}_{s} R-\left(|\alpha|+\frac{1}{2}\right) \frac{\pi}{2}+\eta\left(\tilde{k}_{s}\right)\right]\right| \leq \sin \epsilon, \quad \text { if } s \geq s_{0}
$$

In other words, if $s \geq s_{0}$,

$$
\tilde{k}_{s} R+\eta\left(\tilde{k}_{s}\right)=s \pi+\left(|\alpha|-\frac{1}{2}\right) \frac{\pi}{2}+O(\epsilon) .
$$

Now we split the summation into two parts $\sum_{s=0}^{\infty}=\sum_{s=0}^{s_{0}-1}+\sum_{s=s_{0}}^{\infty}$. The first term will vanish eventually as $R \rightarrow \infty$ and so the second term dominates the whole summation. For $s \geq s_{0}$, we may approximate $\tilde{k}_{s}$ 's in the summation by (19) (omitting $O(\epsilon)$ term) and the error of the approximate sum is easily seen to be $O\left(\epsilon \cdot R^{0}\right)$, which vanishes as $\epsilon \rightarrow 0$ and $R \rightarrow \infty$. This allows us to replace $\tilde{k}_{s}$ by a function $\tilde{k}(s)$ defined for real $s$ as

$$
\tilde{k}(s) R+\eta(\tilde{k}(s))=s \pi+\left(\alpha-\frac{1}{2}\right) \frac{\pi}{2},
$$


when $s \geq s_{0}$. [k(s) is defined similarly.] Then by use of Euler-Maclaurin formula [20, the summation may be converted into an integral,

$$
\sum_{s=0}^{\infty}\left[e^{-\frac{\beta \tilde{k}_{s}^{2}}{m}}-e^{-\frac{\beta k_{0, s}^{2}}{m}}\right]=\int_{s_{0}}^{\infty} d s\left[e^{-\frac{\beta \tilde{k}^{2}(s)}{m}}-e^{-\frac{\beta k^{2}(s)}{m}}\right]+O\left(\epsilon \cdot R^{0}\right)+O(1 / R),
$$

where we did not write explicitly other terms in the Euler-Maclaurin formula which are $O(1 / R)$ at most. From (20), $\frac{d s}{d \tilde{k}}=\frac{1}{\pi}\left(R+\frac{d \eta}{d \tilde{k}}\right)$ and $\frac{d s}{d k}=\frac{R}{\pi}$. Thus we get?

$$
\lim _{R \rightarrow \infty} \sum_{s=0}^{\infty}\left[e^{-\frac{\beta \tilde{k}_{s}^{2}}{m}}-e^{-\frac{\beta k_{s}^{2}}{m}}\right]=\frac{1}{\pi} \int_{0}^{\infty} d k \frac{d \eta}{d k} e^{-\frac{\beta k^{2}}{m}}+\frac{1}{\pi} \eta(0) .
$$

From (16), $\eta(0)=0$ unless $\kappa=0$ and so we drop this term from now on assuming $\kappa \neq 0$. $\frac{d \eta}{d k}$ can easily be computed from (16). Inserting this into (22), we finally obtain

$$
B(T)=B_{0}(T)-2 \lambda_{T}^{2}\left\{e^{\varepsilon} \theta(-\sigma)+\frac{|\alpha| \sigma}{\pi} \sin \pi|\alpha| \int_{0}^{\infty} \frac{d t e^{-\varepsilon t} t^{|\alpha|-1}}{1+2 \sigma \cos \pi \alpha t^{|\alpha|}+t^{2|\alpha|}}\right\}
$$

where $\varepsilon=\beta \kappa^{2} / m$. If we put $\sigma=-1$, this result is equivalent to the formulas in refs. [16,[17].

The above integration cannot be performed analytically in general. In case of semions, $|\alpha|=1 / 2$, it is reduced to the error integral,

$$
B(|\alpha|=1 / 2, T)=B_{0}(|\alpha|=1 / 2, T)-\lambda_{T}^{2} e^{\varepsilon}[1-\sigma \operatorname{Erf}(\sqrt{\varepsilon})],
$$

where $\operatorname{Erf}(x)=\frac{2}{\sqrt{\pi}} \int_{0}^{x} e^{-t^{2}} d t$. Compared with $B_{0}(|\alpha|=1 / 2, T)=\lambda_{T}^{2} / 8$, the second term becomes dominant for most of $\varepsilon$ except near the hard-core limit $\varepsilon \rightarrow \infty$ with $\sigma=+1$.

For general value of $\alpha$, one can obtain various limiting behaviors by examining the integral. First, for $\varepsilon \gg 1$, we find the asymptotic behavior

$$
B(T)=B_{0}(T)-2 \lambda_{T}^{2}\left[e^{\varepsilon} \theta(-\sigma)+\frac{\sigma}{\pi} \frac{\Gamma(|\alpha|+1)}{\varepsilon^{|\alpha|}} \sin \pi|\alpha|+\cdots\right] .
$$

We see that, for $\sigma=+1$, the hard-core result is recovered but, if $\sigma=-1$, the bound state contribution dominates the virial coefficient. Because of the nature of the virial expansion,

\footnotetext{
${ }^{2}$ With the second term $\frac{1}{\pi} \eta(0)$, (22) generalizes the usual formula in terms of phase shifts 21] whose naive application does not produce the correct result in anyon case as noted in 13.

${ }^{3}$ When $\kappa=0, \eta=\pi|\alpha|$ and the first term involving $\frac{d \eta}{d k}$ becomes zero.
} 
(25) is physically meaningful only for parameters in the region $\kappa^{2} / m \gg k_{B} T \gg \rho / m$ where $\rho$ is the particle number density. For $\varepsilon \ll 1$, which corresponds to high-temperature limit, we find after some calculation

$$
\begin{aligned}
B(T) & =B_{0}(T)-2 \lambda_{T}^{2}|\alpha|\left(1-\sigma \varepsilon^{|\alpha|}+\cdots\right), \\
& =\lambda_{T}^{2}\left(-\frac{1}{4}-|\alpha|-\frac{\alpha^{2}}{2}\right)+2 \sigma \lambda_{T}^{2}|\alpha| \varepsilon^{|\alpha|}+\cdots,
\end{aligned}
$$

which differs from the hard-core result by $-2 \lambda_{T}^{2}|\alpha|$ for $\varepsilon=0$.

One may also consider the behavior of $B(T)$ near the bosonic $(\alpha=0)$ and the fermionic $(|\alpha|=1)$ points. After some calculation, we obtainf, for $\alpha \approx 0$ and for nonzero finite $\varepsilon$,

$$
\begin{aligned}
B(T) & =B_{0}(T)-2 \lambda_{T}^{2} \nu(\varepsilon) \theta(-\sigma)-|\alpha| \lambda_{T}^{2}+O\left(\alpha^{2}\right) \\
& =-\left[\frac{1}{4}+2 \nu(\varepsilon) \theta(-\sigma)\right] \lambda_{T}^{2}+O\left(\alpha^{2}\right)
\end{aligned}
$$

where $\nu(\varepsilon)$ is the Neumann function defined by [22]

$$
\nu(\varepsilon)=\int_{0}^{\infty} \frac{\varepsilon^{t} d t}{\Gamma(t+1)} .
$$

Therefore the linear terms in $|\alpha|$ are cancelled out, i.e., $B(T)$ is a smooth function of $\alpha$ near $\alpha=0$ and the cusp at the bosonic point existing for hard-core anyons disappears. On the other hand, near the fermionic point, we find

$$
\begin{aligned}
B(T) & =B_{0}(T)-2 \lambda_{T}^{2} e^{-\sigma \varepsilon}+f_{\sigma}(\varepsilon)(1-|\alpha|)+\cdots \\
& =\lambda_{T}^{2}\left[-\frac{1}{2}(1-|\alpha|)^{2}+\frac{1}{4}-2 e^{-\sigma \varepsilon}\right]+f_{\sigma}(\varepsilon)(1-|\alpha|)+\cdots,
\end{aligned}
$$

where $f_{\sigma}(\varepsilon)(\sigma= \pm 1)$ are some complicated functions of $\varepsilon$ involving exponential integrals whose explicit forms are not much illuminating. Thus we see that in general a cusp exists at the fermionic point $|\alpha|=1$ for anyons without hard core.

\footnotetext{
${ }^{4}$ When $\sigma=-1$ and $\alpha=0$ this result gives the second virial coefficient of bosons with the selfadjoint extension discussed in [6,17].
} 
The physical reason for this change of the cusp position may be the following. For the hard-core boundary condition, anyons behave more like fermions in the sense that they cannot overlap and the lack of hard core for bosons is responsible for the cusp [14]. In contrast, for other boundary conditions, anyons can overlap like bosons and this generates a cusp at the fermionic point where particles cannot overlap.

We plot these results in Figure 1. The deviation of the virial coefficient from hard-core result is quite significant for reasonable values of $\varepsilon=\beta \frac{\kappa^{2}}{m}$. Also, Figure 1 clearly shows cusps at $|\alpha|=1$ and smoothness of curves at $\alpha=0$.

One may be able to generalize this calculation to a system of multi-species of anyons obeying mutual statistics [23]. This system is known to be related with multi-layered quantum Hall effect. In this case self-adjoint extension is allowed in $p$-wave part as well as in $s$-wave part and it introduces another scale parameter. Field theoretically it would correspond to introducing a nonrenormalizable contact interaction with derivatives of delta functions. It may be interesting to see the effect of these scale parameters on various physical properties of the system. Also, the analysis of this paper may be able to be generalized to a system of non-Abelian Chern-Simons particles for which the second virial coefficient was recently calculated in the hard-core case [24].

We would like to thank Prof. C. Lee for bringing my attention to this topic and for continuous encouragement, and S. J. Kim for useful discussions. We also thank A. Moroz and R. Soldati for informing us of their works. This work was supported by the Korea Science and Engineering Foundation through the SRC program. 


\section{REFERENCES}

[1] J. M. Leinaas and J. Myrhein, Nuovo Cimento B 37 (1977) 1; G. A. Goldin, R. Menikoff, and D. H. Sharp, J. Math. Phys. 21 (1980) 21; F. Wilczek, Phys. Rev. Lett. 48 (1982) 1144; for a review, see F. Wilczek, Fractional Statistics and Anyonic Superconductivity (World Scientific, 1990).

[2] S. Albeverio, F. Gesztesy, R. Høegh-Krohn, and H. Holden, Solvable Models in Quantum Mechanics (Springer-Veerlag, Berlin, 1988).

[3] Y. Aharonov and D. Bohm, Phys. Rev. 115 (1959) 485.

[4] C. R. Hagen, Phys. Rev. D31 (1985) 848.

[5] R. Jackiw and S. Y. Pi, Phys. Rev. D42 (1990) 3500.

[6] R. Jackiw, in M. A. Bég Memorial Volume, edited by A. Ali and P. Hoodbhoy (World Scientific, Singapore, 1991); O. Bergman and G. Lozano, Ann. Phys. (N. Y.) 229 (1994) 416.

[7] G. Amelino-Camelia, Phys. Lett. 326B (1994) 282; C. Manuel and R. Tarrach, Phys. Lett. 328B (1994) 113; S. Ouvry, Phys. Rev. D50 (1994) 5296; G. Amelino-Camelia, Phys. Rev. D51 (1995) 2000.

[8] G. Amelino-Camelia and D. Bak, Phys. Lett. 343B (1995) 231.

[9] S. J. Kim Phys. Lett. 343B (1995) 244; S. J. Kim and C. Lee Phys. Rev. D55 (1997) 2227.

[10] M. Bourdeau and R. D. Sorkin, Phys. Rev. D45 (1992) 687.

[11] D. Bak and O. Bergman, Phys. Rev. D51 (1995) 1994.

[12] D. P. Arovas, R. Schrieffer, F. Wilczek and A. Zee, Nucl. Phys. B251 (1985) 117.

[13] A. Comtet, Y.Georgelin and S. Ouvry, J. Phys. A22 (1989) 3917. 
[14] D. Loss and Y. Fu, Phys. Rev. Lett. 67 (1991) 294.

[15] See also T. Blum, C. R. Hagen and S. Ramaswamy, Phys. Rev. Lett. 64 (1990) 709; B. Kahng and K. Park, Phys. Rev. B45 (1991) 8158.

[16] A. Moroz, Phys. Rev. A53 (1996) 669.

[17] P. Giacconi, F. Maltoni and R. Soldati, Phys. Rev. B53 (1996) 10065.

[18] See for example K. Huang, Statistical Mechanics (John Wiley and Sones, 1963).

[19] C. Manuel and R. Tarrach, Phys. Lett. 268B (1991) 222.

[20] M. Abramowitz and I. Stegun, Handbook of Mathematical Functions (Dover, 1972).

[21] E. Bethe and G. E. Ulenbeck, Physica 4 (1937) 915.

[22] I. S. Gradstein and I. M. Ryzik, Tables of integrals, series and products (Academic Press, 1980).

[23] X. G. Wen and A. Zee, Nucl. Phys. B15 (1990) 135; Phys. Rev. B44 (1991) 274; B. Blok and X. G. Wen, Phys. Rev. B43 (1991) 8337; F. Wilczek, Phys. Rev. Lett. 69 (1992) 132; C. R. Hagen Phys. Rev. Lett. 68 (1992) 3821; C. Kim, C. Lee, P. Ko, B.-H. Lee, and H. Min, Phys. Rev. D48 (1993)1821; D. Wesolowski, Y. Hosotani, and C.-L. Ho, Int. J. Mod. Phys. A9 (1994) 969.

[24] T. Lee, Phys. Rev. Lett. 74 (1995) 4967. 


\section{FIGURES}

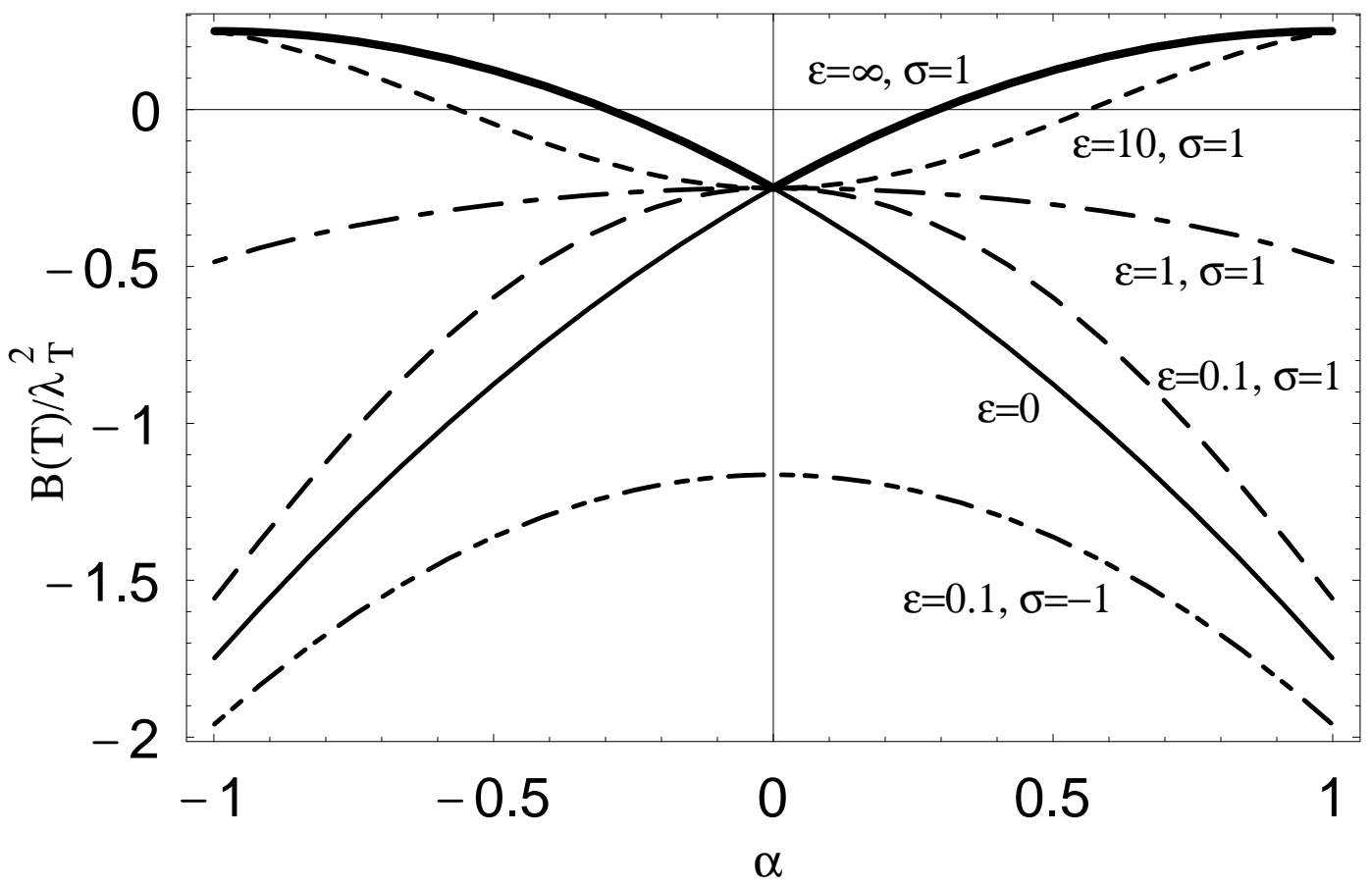

FIG. 1. The second virial coefficient of anyons without hard core as functions of the statistical parameter $\alpha$ for various values of $\varepsilon=\beta \kappa^{2} / m . \varepsilon=\infty$ with $\sigma=1$ corresponds to hard-core limit and $\varepsilon=0$, high-temperature limit. 